\title{
Entropy of electrolytes
}

\author{
Brian B. Laird \\ Department of Chemistry, University of Kansas, Lawrence, Kansas 66045
}

A. D. J. Haymet ${ }^{\text {a) }}$

School of Chemistry, University of Sydney, NSW 2006, Australia

(Received 4 October 1993; accepted 19 November 1993)

\begin{abstract}
The entropy of 1-1 and 2-2 model electrolytes is calculated from an expansion in terms of the multiparticle correlation functions. For electrolytes, a simple truncation of this expansion is never sufficient for the accurate calculation of the entropy, even in the limit of low concentration, in marked contrast to the behavior for short-ranged potentials. However, a partial, but infinite-order, summation of the expansion is shown to yield both the correct low-concentration limit and excellent results over a wide range of concentrations for both 1-1 and 2-2 electrolytes. The consequences of this result for some earlier applications of the entropy expansion to electrolytes are discussed.
\end{abstract}

\section{ELECTROLYTES}

The prediction of the properties of electrolyte solutions poses a particular challenge to liquid theory, due to the long-range nature of the Coulomb forces and the requirements of charge neutrality. ${ }^{1}$ The popular Debye-Hückel (DH) approximation provides some insight, but it is of practical use only for very dilute aqueous solutions of univalent ions (1-1 electrolytes). Efforts to go beyond the DH level usually involve integral equations, such as the hypernetted chain (HNC) approximation. Although generally successful for 1-1 electrolytes, the HNC approximation yields unphysical solutions for dilute solutions of divalent ions (2-2 electrolytes). ${ }^{2,3}$ Recently, Duh and Haymet ${ }^{4}$ have developed an approximation which overcomes the aforementioned deficiencies of the HNC closure, and which yields accurate results for the 2-2 electrolyte over the full concentration range. The mean spherical approximation (MSA) has different but similar limitations. ${ }^{5}$

Computer simulations yield exact information about the structure of a liquid at a fixed thermodynamic state through the calculation of the pair correlation functions $g_{\alpha \beta}(r)$, and integral equations yield approximations to these functions. For model electrolytes with only pairwiseadditive interactions between ions, some thermodynamic quantities, such as the average potential energy and the pressure, may be calculated directly from these pair correlation functions. However, for other important quantities, such as the entropy and free energy, such a direct and exact calculation is not possible. Traditionally, such quantities have been calculated indirectly using the technique of thermodynamic integration, which involves the calculation of the properties of the solution over an entire range of thermodynamic states between a reference (ideal) state and the state of interest, and this often requires significant computer resources.

A direct, but approximate, method for calculating the entropy (and hence the free energy) from the pair correlations alone has been explored for a variety of liquids. ${ }^{6-12}$

"Author to whom correspondence should be addressed.
This method is based on truncation of an exact, but infinite-order, expansion for the entropy in terms of multiparticle correlation functions. In this paper, this exploration is continued for electrolytes. Comparison is made with a similar study of a hard-core 1-1 electrolyte by Hummer and Soumpasis. ${ }^{13}$

Our general argument applies to any model of an electrolyte, since it appeals to correlation functions. To be definite, in this paper we will use correlation functions generated for a particular electrolyte model, a fluid of symmetric, charged soft spheres introduced by Rossky and co-workers. ${ }^{14}$ The interaction potential between ion species $\alpha$ and $\beta$ is given by

$$
\phi_{\alpha \beta}(r)=\frac{k B}{\sigma}\left(\frac{\sigma}{r}\right)^{9}+\frac{Z_{\alpha} Z_{\beta} e^{2}}{\epsilon r},
$$

where $Z_{\alpha}$ is the number of electron charges on ion type $\alpha$. The solvent is modeled as a structureless continuum with dielectric constant $\epsilon$, here taken to be that of water at $25^{\circ} \mathrm{C}, \epsilon=78.358 \epsilon_{0}$. The energy parameter $B$ and the ionic diameter $\sigma$ are set equal to $5377.75\left|Z_{\alpha} Z_{\alpha}\right| \AA \mathrm{K}$ and $2.8428 \AA$, respectively. These values are chosen to facilitate comparison with earlier work. Note that the cation and anion are assumed to be of equal size. This potential is similar to the standard restricted primitive model, except the ion hard core has been replaced by a more realistic soft repulsion. In all calculations reported here, the temperature is $25^{\circ} \mathrm{C}$.

For this paper we require accurate correlation functions for the electrolyte. Our analysis does not depend on the source of these correlation functions. For example they could be obtained from computer simulations at each concentration. For simplicity, all structural data for this study are generated using the electrolyte integral-equation theory of Duh and Haymet, ${ }^{4}$ which includes an approximate bridge function. This closure yields highly accurate results for both 1-1 and 2-2 electrolytes at $25^{\circ} \mathrm{C}$, over the wide concentration range $c=0.001$ to $1 \mathrm{M}\left(1 \mathrm{M}=1 \mathrm{~mol} \mathrm{dm}^{-3}\right)$. Full details of the method are presented in the original paper. ${ }^{4}$ The results for the 2-2 electrolyte at low concen- 
trations exhibit none of the pathologies of the HNC approximation. This makes possible the study of very low concentrations, which is crucial for determining the concentrations above which the Debye-Hückel approximation breaks down for the 2-2 electrolyte.

In Sec. II we review the entropy expansion in correlation functions and present results for electrolytes in Sec. III. Comparison with earlier calculations of the entropy of an electrolyte are present in Sec. IV, and our conclusions are collected in Sec. V.

\section{THERMODYNAMICS FROM ENTROPY EXPANSIONS}

As mentioned above, some thermodynamic quantities cannot be calculated directly from the pair correlation functions. The major drawback to the traditional thermodynamic integration method for calculating the free energy is that it is indirect; the free energy cannot be calculated simply from the structural properties at the thermodynamic state of interest, but rather requires information about all thermodynamic states along some path connecting the state with a convenient (ideal) reference state. Such additional information is often inconvenient, timeconsuming or even impossible to obtain. For this reason, the development of accurate direct methods has been of much recent interest. ${ }^{6-8,10-12}$

One such direct method involves the use of an expansion for the entropy in terms of multiparticle correlation functions. For a multicomponent liquid with pairwiseadditive interparticle potential energies, the average potential energy may be written exactly as a functional of those potentials and the pair correlation functions $g_{\alpha \beta}(r)$, where $\alpha$ and $\beta$ index particle species. However, a calculation of the free energy requires a knowledge of both the entropy and the energy. Unfortunately, even for pairwise-additive systems, the expansion of the entropy in terms of multiparticle correlation functions does not truncate at the pair level, but instead has contributions from all orders. ${ }^{15,16}$ At present the calculation of multiparticle correlation functions is at best an extremely difficult task, and recent work has focused on manipulating the entropy expansion in order to obtain the best possible estimate of the entropy from the pair correlation functions alone. ${ }^{10}$

Building upon the work of Kirkwood, ${ }^{17}$ Green, ${ }^{15}$ Nettleton and Green, ${ }^{16}$ and Raveché, ${ }^{18}$ Hernando ${ }^{8,9}$ recast the entropy expansion into a particularly useful form, via a partial resummation of the higher-order terms. Generalized to a mixture of $v$ components by Laird, Wang, and Haymet, ${ }^{19}$ the resulting expansion can be written as the sum of four terms,

$S\left[g^{(n)}\right] / N k=s_{\text {ideal }}+s^{(2)}+s_{\text {ring }}+\sum_{i=3}^{\infty} s_{\text {mix }}^{\prime}(i)\left[g_{\alpha \beta}^{(m)} ; m \leqslant i\right]$.

The functions $g_{\alpha \beta \ldots}^{(m)}\left(\mathbf{r}_{\alpha}, \mathbf{r}_{\beta}, \ldots\right)$ are the $m$-particle (multicomponent) correlation functions of the liquid. In the ensemble invariant form, ${ }^{6}$ the first term

$$
s_{\text {ideal }} \equiv=\frac{5}{2}-\sum_{\alpha=1}^{\nu} x_{\alpha} \ln \rho_{\alpha} \Lambda_{\alpha}^{3}
$$

is the ideal gas contribution, where $\alpha$ is the species label, $\Lambda_{\alpha}$ is the de Broglie thermal wavelength, $\rho_{\alpha}$ is the partial number density, $\rho=\Sigma_{\alpha=1}^{v} \rho_{\alpha}$ is the total number density, and $x_{\alpha}=\rho_{\alpha} / \rho$ is the mole fraction. The second term,

$$
\begin{aligned}
s^{(2)} \equiv & -\frac{\rho}{2} \sum_{\alpha=1}^{\nu} \sum_{\beta=1}^{v} x_{\alpha} x_{\beta} \int d r_{\alpha \beta}\left[g_{\alpha \beta}^{(2)}\left(r_{\alpha \beta}\right) \ln g_{\alpha \beta}^{(2)}\left(r_{\alpha \beta}\right)\right. \\
& \left.-g_{\alpha \beta}^{(2)}\left(r_{\alpha \beta}\right)+1\right],
\end{aligned}
$$

results from the usual second-order truncation of the entropy expansion, and contains the familar " $g \ln g$ " term. The next term is the so-called "ring" contribution,

$$
\begin{aligned}
s_{\text {ring }} \equiv & \frac{1}{2(2 \pi \rho)^{3}} \int d \mathbf{k}\left[\ln |\mathbf{I}+\widetilde{\mathbf{H}}(k)|+\frac{1}{2} \operatorname{Tr} \widetilde{\mathbf{H}}^{2}(k)\right. \\
& -\operatorname{Tr} \widetilde{\mathbf{H}}(k)]
\end{aligned}
$$

where $I$ is the $v \times v$ identity matrix and

$$
\widetilde{\mathbf{H}}(k)_{\alpha \beta} \equiv \rho_{\alpha}^{1 / 2} \widetilde{h}_{\alpha \beta}(k) \rho_{\beta}^{1 / 2} .
$$

Hernando derived this term by extracting the leading contribution ("ring" diagram) from each individual order of the expansion hierarchy, and summing these contributions to infinite order. The function $\widetilde{h}_{\alpha \beta}(k)$ is the Fourier transform of the total correlation function $h_{\alpha \beta}(r) \equiv g_{\alpha \beta}(r)-1$. Note that when pair correlation functions obtained from the HNC approximation are inserted into this expansion, the sum of the first three terms is exactly equal to the well-known HNC entropy.

The first three terms require structural information only up to pair correlation functions. The remaining terms, $s_{\text {mix }}^{\prime}(i)$, result from the subtraction of the "ring" terms from the higher-order $(i \geqslant 3)$ terms in the expansion, and they contain contributions from all multiparticle correlation functions up to order $i$. For a given order of expansion, these terms are higher order in the density than the corresponding "ring" term, and to a good approximation they may be neglected in the low to moderate density regime $e^{10}$ of simple liquids. Our goal here is to explore this expansion for electrolytes.

\section{RESULTS FOR ELECTROLYTES}

As a function of concentration, we have calculated $s^{(2)}$ and $s^{(2)}+s_{\text {ring }}$ (hereafter referred to as the ring approximation) for both 1-1 and 2-2 electrolytes at $25^{\circ} \mathrm{C}$, using structural data obtained for the model electrolyte model using the integral equation described above. For comparison, the "exact" excess entropy for these electrolytes has been calculated using thermodynamic integration of the data from the integral equation from the $\rho=0$ zero concentration limit. The results of these calculations for the 1-1 electrolyte up to the concentration $9.0 \mathrm{M}$ are shown in Fig. 1, and in Fig. 2 for the 2-2 electrolyte up to $2.25 \mathrm{M}$. 


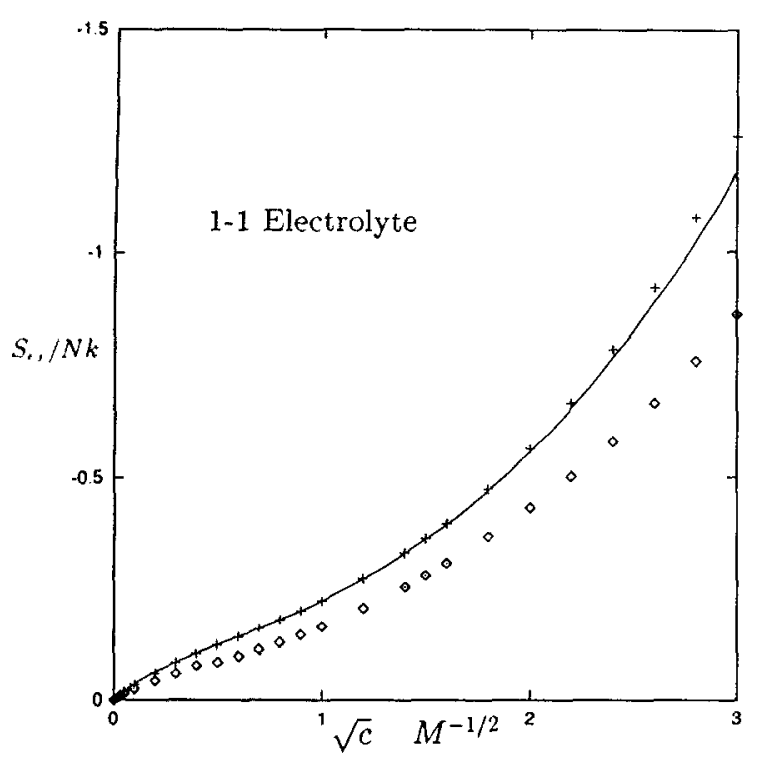

FIG. 1. Excess entropy per particle at $25^{\circ} \mathrm{C}$ for the model 1-1 electrolyte described in the text, as a function of the square root of the molar concentration. Three methods of calculation are shown, thermodynamic integration from the $c=0$ state (solid line); the entropy expansion truncated at second order, $s^{(2)}$ (diamonds); and the same entropy expansion with the "ring" term added, $s^{(2)}+s_{\text {ring }}$ (crosses).

For both the 1-1 and 2-2 electrolytes, Figs. 1 and 2 show that the ring approximation is extremely accurate over the concentration ranges studied. Note that the entropy of the 2-2 electrolyte is qualitatively different than the 1-1 electrolyte entropy. In both cases, the second-order term $s^{(2)}$ is a poor approximation to the entropy. This is to be expected, since the ring approximation for the entropy is identical to that obtained from the HNC closure, which is known to be very accurate for electrolytes. ${ }^{19,20}$ A third approximation, the so-called "incompressible" approxima-

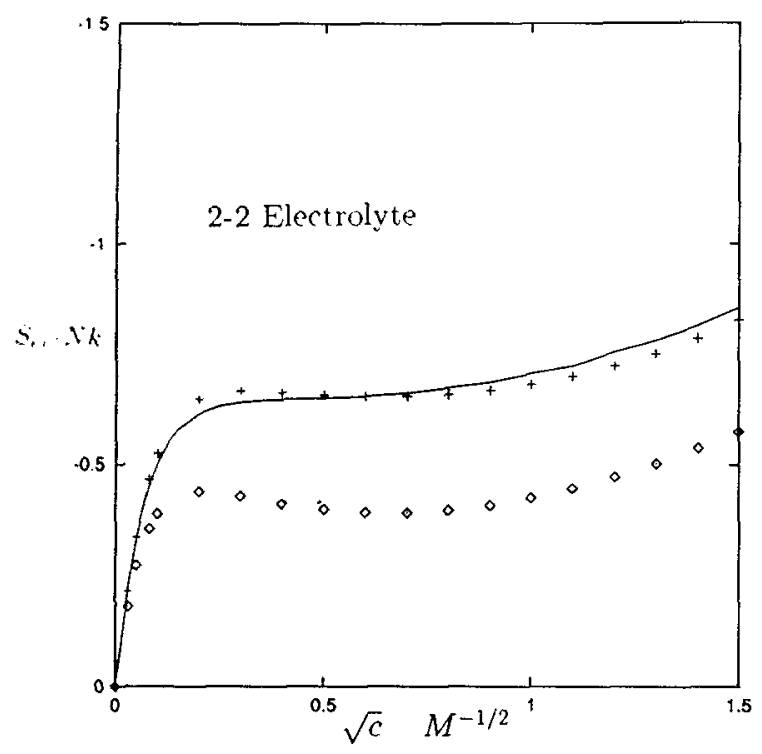

FIG. 2. Same as Fig. 1 for the 2-2 electrolyte. tion developed recently ${ }^{10}$ has not been considered here, because even at these seemingly high concentrations, the actual number density of the electrolyte species is quite low, leading to compressibilities that are too high for this approximation to be valid.

\section{COMPARISON WITH THE DEBYE-HÜCKEL APPROXIMATION}

At sufficiently low concentrations (low ionic strengths), the properties of an electrolyte are governed by the so-called Debye-Hückel limiting law, in which the pair correlation function is given by

$$
\ln g_{\alpha \beta}(r) \rightarrow-\frac{Z_{\alpha} Z_{\beta} e^{2} e^{\kappa r}}{k T \epsilon r},
$$

where the inverse Debye length $\kappa$ is defined from

$$
\kappa^{2} \equiv \frac{4 \pi e^{2}}{k T \epsilon} \sum_{\alpha=1}^{v} Z_{\alpha}^{2} \rho_{\alpha}
$$

Using thermodynamic integration, it can be shown ${ }^{21}$ that, in this limit, the excess entropy per particle varies as

$$
s \approx s_{\mathrm{DH}}=-\frac{\kappa^{3}}{24 \pi \rho} .
$$

It is useful to examine the behavior of the entropy expansion approximations in this limit. For the second-order truncation $s^{(2)}$, substituting the $\mathrm{DH}$ correlation function [Eq. (7)] into Eq. (4) yields

$$
s^{(2)} \approx-\frac{\kappa^{3}}{32 \pi \rho} \text {. }
$$

Comparison of Eqs. (9) and (10) shows that $s^{(2)}$ approaches the zero-concentration limit with the incorrect limiting slope! This is a consequence of the long-ranged nature of the Coulomb potential. In liquids with shortranged potentials, the virial series is analytic at $\rho=0,{ }^{10}$ and there is no difficulty with this limit.

The limit of the ring approximation $s_{\text {ring }}$ yields

$$
s_{\text {ring }} \approx-\frac{\kappa^{3}}{8 \pi \rho} \sum_{i=3}^{\infty} \frac{(2 n-5) ! !}{n(2 n-2) ! !}=-\frac{\kappa^{3}}{96 \pi \rho} .
$$

The addition of Eqs. (10) and (11) recovers the correct low concentration limit [Eq. (9)].

To illustrate these limits, Figs. 3 and 4 display $s^{(2)}$, the ring approximation $s^{(2)}+s_{\text {ring }}$, and the thermodynamic integration result as a function of $c^{1 / 2}$ (at $25^{\circ} \mathrm{C}$ ) at very low concentrations for the 1-1 and 2-2 electrolytes. The limiting slopes are also shown. The ring limit slopes are clearly correct. In addition, note that for the 2-2 electrolyte the entropy attains the DH limiting law only for concentrations below $10^{-7} \mathrm{M}$ !

Hence, the entropy of an electrolyte cannot be approximated by a simple finite sum of two-body, or three-body terms. The simple pair entropy yields a limiting slope incorrect by $25 \%$. Some evidence of this arose previousiy in a computer simulation of the restricted primitive model of a 1-1 electrolyte ( $\mathrm{NaCl}$ ) by Hummer and Soumpasis. ${ }^{13}$ Using thermodynamic integration of the computer simula- 


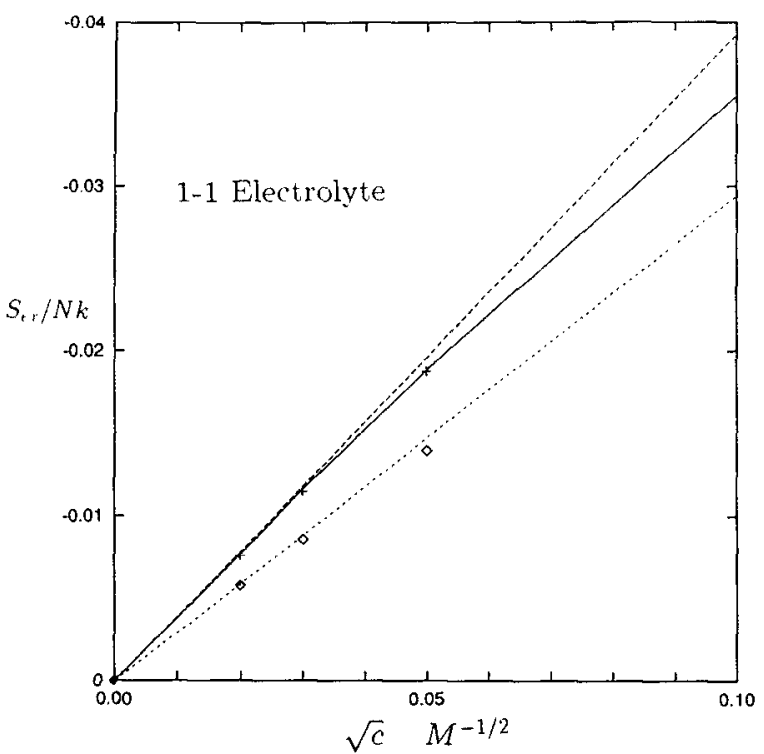

FIG. 3. Excess entropy per particle at $25^{\circ} \mathrm{C}$ and low concentration for the $1-1$ electrolyte as a function of the square root of the molar concentration. Three methods of calculation are shown: thermodynamic integration from the $c=0$ state (solid line); the entropy expansion truncated at second order $s^{(2)}$ (diamonds); and the same entropy expansion with the "ring" term added, $s^{(2)}+s_{\text {ring }}$ (crosses). The limiting slopes for the $s^{(2)}$ truncation (short dashed line) and the Debye-Hückel model (long dashed line) are also shown.

tion data to obtain the "exact" entropy, they showed that neither a second- nor third-order truncation of the entropy expansion yields correct results for intermediate densities. Since data for very low concentrations are not published, one cannot obtain information about these approximations in the extremely dilute limit.

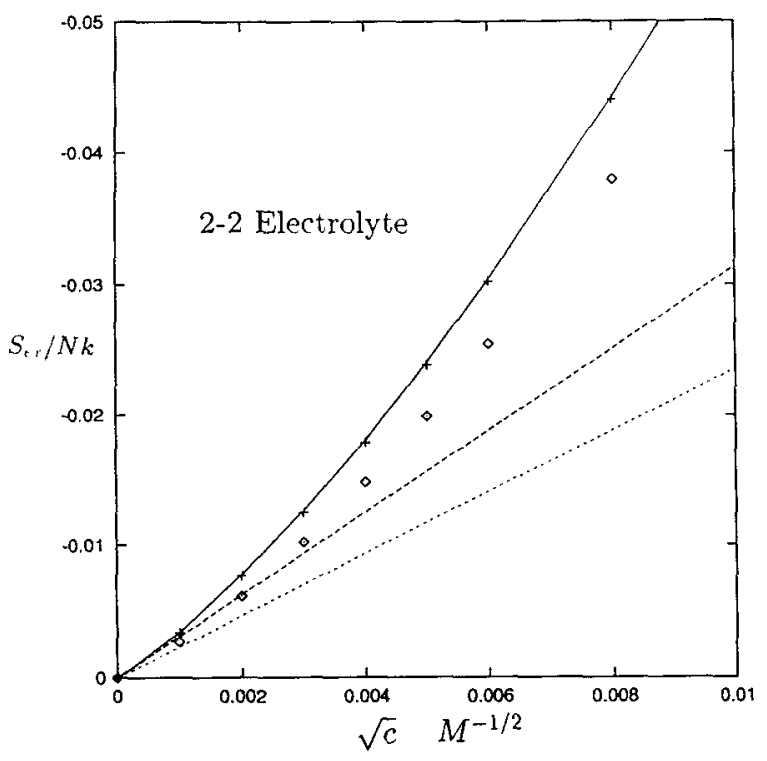

FIG. 4. Same as Fig. 3 for the 2-2 electrolyte.

\section{v. CONCLUSIONS}

We have shown that the entropy of an electrolyte cannot be described by simple, finite-order truncations of an expansion in terms of multiparticle correlation functions, even at very low concentrations (Debye-Hückel limit). This differs from liquids with short-range potential energies.

Our results prove that it is never correct to omit the "ring" term [Eq. (11)] to the entropy expansion when studying systems with long-range, Coulombic interactions. Therefore the results of electrolyte studies that use only a low-order truncation, such as the calculation of the free energy for the electrical double layer, ${ }^{22}$ must be treated with caution.

In addition, we show that the entropy of a 2-2 electrolyte at $25^{\circ} \mathrm{C}$ approaches the Debye-Hückel limiting value only for concentrations below $10^{-7} \mathrm{M}$. It is well known that the Debye-Hückel approximation breaks down for dilute 2-2 electrolytes, but the difficulty of doing computer simulations at very low concentrations, combined with the fact that most integral equations (such as the HNC) fail to yield physical solutions for dilute 2-2 electrolytes ${ }^{3}$ has prevented previous efforts to locate precisely the concentration at which the breakdown occurs.

\section{ACKNOWLEDGMENTS}

This research was supported by the Australian Research Council (ARC) (Grant No. A29131271). We thank Der-Ming Duh (Utah) for use of the electrolyte integral-equation code, ${ }^{4}$ and Michael Booth and Dr. Anna Nyberg for a compact proof of the infinite summation in Eq. (11). For providing a stimulating research environment for part of the work presented here, B. B. L. wishes to thank the Department of Chemistry of the University of Wisconsin-Madison. A. D. J. H. acknowledges a Visiting Fellowship at the ANU Research School of Chemistry, where this work was completed.

${ }^{1}$ J. P. Hansen and I. R. McDonald, Theory of Simple Liquids, 2nd ed. (Academic, New York, 1986).

${ }^{2}$ Yu. V. Kalyuzhnyi, M. F. Holovko, and A. D. J. Haymet, J. Chem. Phys. 95, 9151 (1991).

${ }^{3}$ J. S. Høye, E. Lomba, and G. Stell, Mol. Phys. 79, 523 (1993).

${ }^{4}$ D.-M. Duh and A. D. J. Haymet, J. Chem. Phys. 97, 7716 (1992).

${ }^{5}$ M. J. Gillan, Mol. Phys. 41, 75 (1980).

${ }^{6}$ A. Baranyai and D. J. Evans, Phys. Rev. A 40, 3817 (1989).

${ }^{7}$ D. C. Wallace, J. Chem. Phys. 87, 4843 (1987).

${ }^{8} \mathrm{~J}$. A. Hernando, Mol. Phys. 69, 319 (1990).

9 J. A. Hernando, Mol. Phys. 69, 327 (1990).

${ }^{10}$ B. B. Laird and A. D. J. Haymet, Phys. Rev. A 45, 5680 (1992).

${ }^{11}$ B. B. Laird and A. D. J. Haymet, J. Chem. Phys. 97, 2153 (1992).

${ }^{12}$ L. Lee, J. Chem. Phys. 97, 8606 (1992).

${ }^{13}$ G. Hummer and D. M. Soumpasis, J. Chem. Phys. 98, 581 (1993).

${ }^{14}$ P. J. Rossky, J. B. Duduwicz, B. L. Tembe, and H. L. Friedman, J. Chem. Phys. 73, 3372 (1980).

${ }^{15} \mathrm{H}$. S. Green, The Molecular Theory of Fluids (North-Holland, Amsterdam, 1952).

${ }^{16}$ R. E. Nettleton and M. S. Green, J. Chem. Phys. 29, 1365 (1958).

${ }^{17}$ J. G. Kirkwood, J. Chem. Phys. 10, 394 (1942). 
${ }^{18} \mathrm{H}$. J. Raveché, J. Chem. Phys. 55, 2242 (1971).

${ }^{19}$ B. B. Laird, J. Wang, and A. D. J. Haymet, Phys. Rev. E 47, 2491 (1993); 48, 4145 (1993).

${ }^{20}$ Note that the ring approximation is derived from an entropy expansion without consideration of the approximation used to calculate the cor- relation functions, so the use of correlation functions obtained from other than HNC closures, as done here, is justified.

${ }^{21}$ D. A. McQuarrie, Statistical Mechanics (Harper and Row, New York, 1976).

${ }^{22}$ J. Th. G. Overbeek, Colloids and Surf. 51, 61 (1990). 